\title{
Macouria - La Retraite, Domaine des Roches
}

\section{Rouges}

Opération préventive de diagnostic (2015)

Jérôme Briand

\section{(2) OpenEdition}

Journals

Édition électronique

URL : https://journals.openedition.org/adlfi/16260

ISSN : 2114-0502

Éditeur

Ministère de la Culture

\section{Référence électronique}

Fabrice Casagrande, Jérôme Briand, « Macouria - La Retraite, Domaine des Roches Rouges » [notice archéologique], ADLFI. Archéologie de la France - Informations [En ligne], Espace Caraïbes, mis en ligne le 16 janvier 2016, consulté le 27 juillet 2021. URL : http://journals.openedition.org/adlfi/16260

Ce document a été généré automatiquement le 27 juillet 2021.

(c) ministère de la Culture et de la Communication, CNRS 


\title{
Macouria - La Retraite, Domaine des Roches Rouges
}

\author{
Opération préventive de diagnostic (2015)
}

Jérôme Briand

\section{NOTE DE L'ÉDITEUR}

Organisme porteur de l'opération : Inrap

1 C'est un diagnostic archéologique qui a permis la découverte d'une petite occupation précolombienne datée de la fin du Néoindien et située en arrière des cordons de sable qui jouxtent le littoral guyanais. Les cordons littoraux sont des bandes de terre qui ont favorisé l'implantation humaine des temps reculés à aujourd'hui. Le village de Tonate, commune de Macouria, en est un parfait exemple : il se développe parallèlement à la côte sur l'un de ces phénomènes géologiques. Les maisons ainsi que les infrastructures communales s'égrènent de part et d'autre du principal axe de communication : la route nationale $\mathrm{n}^{\circ} 1$. Une fouille menée au lieu-dit de Sainte-Agathe par Matthieu Hildebrand, en 2009 , nous enseigne que cette occupation contemporaine succède à un village plus ancien, daté lui aussi de la fin du Néoindien. En arrière de cet ensemble, on note une vaste zone humide où criques et canaux serpentent dans une plaine façonnée par l'érosion dont émergent par endroits de petits reliefs de quelques dizaines de mètres de haut seulement. C'est sur une sorte de crête, bordée par un petit canal naturel, que s'étend l'occupation de la Retraite, Domaine des Roches Rouges. En arrière-plan se dressent les mornes de Macouria, dont le plus imposant culmine à $78 \mathrm{~m}$ au-dessus du niveau de la mer.

2 Le diagnostic archéologique s'inscrit dans un vaste projet de constructions de 145 logements individuels au lieu-dit la Retraite. Cette opération préventive a été menée afin de repérer la présence de vestiges archéologiques. Pour cela, des sondages linéaires de type tranchée ont été ouvert à l'aide d'une pelle hydraulique. 
3 Un petit canal naturel qui se jette vers l'extrémité nord de la parcelle diagnostiquée dans la crique de Macouria sépare la parcelle diagnostiquée en deux, créant ainsi un relief légèrement vallonné.

4 Sur le relief le plus proche de la confluence s'étend une imposante nappe de vestiges. On y rencontre de nombreux fragments de poteries, mais aussi quelques haches polies ou d'autres objets lithiques variés. Proche de cette aire de rejet, l'expertise archéologique a également révélé une poterie quasiment entière qui contenait les fragments agencés d'une seconde. Malgré une fouille minutieuse en laboratoire aucun reste humain, même carbonisé, n'a été découvert. Doit-on pour autant exclure l'hypothèse d'un ensemble funéraire?

5 Plus au sud, une fois passée une petite dépression de quelques mètres de profondeur quasiment stérile en objets archéologiques (petit vallon), on se retrouve en présence d'un second relief. Au sommet de celui-ci, a été découverte une concentration de petites structures en creux qui pourraient être des trous de poteau, vestiges d'anciens carbets aujourd'hui disparus. Ces derniers n'ont révélé aucun objet dans le sédiment qui les comble. À ce stade de l'étude, nous émettrons donc une réserve concernant leur datation. Néanmoins, il est important de souligner la présence d'artéfacts précolombiens dans les horizons subjacents.

6 En contrebas de ces petits promontoires qui forment une sorte de ligne de crête, coule le petit canal naturel. La puissance stratigraphique qui peut atteindre parfois plus d'1,50 m est composée de colluvions et d'alluvions sablo/limoneuses plus ou moins riches en micas et parfois de couches riches en matière organique, vestiges d'ancienne tourbe ou de vase. Dans ce contexte, on rencontre parfois la présence de tessons de poteries ou de roches en tous genres. Déposés naturellement pour la plupart, quelquesuns de ces artéfacts peuvent être des traces d'éventuelles activités humaines, telles que le puisage d'eau potable. La baignade ou encore la pêche devaient y être également pratiquées.

7 Au-delà de la rive opposée au petit habitat révélé par le diagnostic, on note dans de petites fosses ou dépressions naturelles ou encore posées à même le sol, des poteries cassées et abandonnées sur place. Ces indices isolés semblent indiquer des activités ponctuelles en forêt ou aux abattis.

8 Un charbon de bois a été envoyé au laboratoire Beta Analytic de Miami afin qu'une datation radiocarbone soit réalisée sur cet échantillon. La date ainsi obtenue est comprise entre 1410 et 1445 ans apr. J.-C. (Cal BP 540 to 505). Si l'on compare cette fourchette chronologique avec les datations faites par Matthieu Hildebrand pour le village de Sainte-Agathe, on constate que les deux occupations sont parfaitement contemporaines. Situés à $2 \mathrm{~km}$ seulement, les amérindiens pouvaient aller d'un site à l'autre par voie navigable. La présence de plusieurs pirogues récentes indique que le lit de la crique de Macouria est encore aujourd'hui ainsi emprunté par nos contemporains. Les terres immergées de l'arrière-pays du village implanté sur les cordons littoraux pouvaient donc être propices à l'implantation d'occupations satellitaires ou saisonnières pour y pratiquer, peut-être, l'horticulture dans des abattis s'immisçant localement dans l'immense forêt tropicale du plateau des Guyanes. 


\section{INDEX}

lieux https://ark.frantiq.fr/ark:/26678/pcrtA9QOB3otnt, https://ark.frantiq.fr/ark:/26678/ pcrtSXtVnWzABE, https://ark.frantiq.fr/ark:/26678/pcrt4AS1AEKdgT, https://ark.frantiq.fr/ ark:/26678/pcrt9aK5fmOuXJ

chronologie https://ark.frantiq.fr/ark:/26678/pcrt9hLpUyQcym, https://ark.frantiq.fr/ark:/ 26678/pcrtil5znJ6Z4o

nature https://ark.frantiq.fr/ark:/26678/pcrtWWQS75V5Bc

Année de l'opération : 2015

sujets https://ark.frantiq.fr/ark:/26678/pcrt1DMOWvDF4j, https://ark.frantiq.fr/ark:/26678/ pcrtxs8Kml8jLw, https://ark.frantiq.fr/ark:/26678/pcrtI9ADjL6wEs, https://ark.frantiq.fr/ark:/ 26678/pcrtSVdwKcS3MI, https://ark.frantiq.fr/ark:/26678/pcrtj3ZcwkArSR, https:// ark.frantiq.fr/ark:/26678/pcrt4N9jrETRto, https://ark.frantiq.fr/ark:/26678/pcrtIzSUofuocv, https://ark.frantiq.fr/ark:/26678/pcrtKJVpuP3AET, https://ark.frantiq.fr/ark:/26678/ pcrtDlzbGxWvTo, https://ark.frantiq.fr/ark:/26678/pcrtbptj4SOA1W

\section{AUTEURS}

\section{JÉRÔME BRIAND}

Inrap 